\title{
Surgical Treatment of Hashimoto's Thyroiditis - Pros and Cons from the Perspective of Clinical Results
}

\author{
Viktor Olexandrovich Shidlovskyi ${ }^{1}$, Olexander Viktorovich Shidlovskyi ${ }^{1}$, Michael I. Sheremet 2,*(D), \\ Larysa Petrivna Sydorchuk ${ }^{3}$, Volodymyr Ivanovich Piatnochka ${ }^{1}$, Vitaliy Vasilyevich Maksymyuk ${ }^{2}$, \\ Volodimir Volodimirovich Tarabanchuk ${ }^{2}$, Yan Viktorovich Gyrla ${ }^{2}$, Nina Petrivna Tkachuk ${ }^{2}$, Oleksandr \\ Vasilyevich Bilookyi ${ }^{2}$, Oleh Havrilovich Harabara ${ }^{2}$ \\ 1 I. Ya. Horbachevskyi Ternopol National Medical University, Ternopil, Ukraine \\ 2 Head of the Department of Family Medicine, Bucovinian State Medical University, Chernivtsi, Ukraine \\ 3 Surgery Department No1 of Bukovinian State Medical University, Ukraine Address: Holovna str., 191, 58018 Chernivtsi, \\ Ukraine \\ * Correspondence: mihay171@gmail.com;
}

Scopus Author ID 57193774935

Received: 15.05.2020; Revised: 9.06.2020; Accepted: 12.06.2020; Published: 15.06.2020

\begin{abstract}
Patients with Hashimoto's thyroiditis have hypothyroidism, goiter, and extrathyroidal lesions. Substitution therapy with thyroxine drugs in some cases is not effective. The work studies the feasibility of using surgical treatment - removal of the active site of autoimmune aggression. To study the effect of thyroidectomy on the quality of life of patients with Hashimoto's thyroiditis with extrathyroidal manifestations. Sixty-one patients with Hashimoto's thyroiditis were examined. Among them 29 patients were treated with drugs (control group) and 32 with surgery (main group). The quality of life was studied using the SF-36 questionnaire. TSH levels within euthyroidism were controlled and the level of antibodies to thyroperoxidase was determined. Two years after the surgical treatment, the studied quality of life indicators was improved significantly. The growth in individual indicators ranged from 34 (role-functioning, conditioned by emotional state) to $57 \%$ (vital activity), and the overall health indicator increased by $52 \%$. The level of antibodies to peroxidase decreased almost to the norm. In the group of patients receiving drug treatment, overall quality of life indicators did not change significantly, but there was a tendency to worsen. The level of antibodies to peroxidase remained at high rates without significant changes. Surgical treatment of patients with Hashimoto's thyroiditis improves the quality of life. The level of antibodies to thyroperoxidase after thyroidectomy is reduced to almost physiologically significant indicators.
\end{abstract}

Keywords: Hashimoto's thyroiditis; quality of life; thyroidectomy.

(C) 2020 by the authors. This article is an open-access article distributed under the terms and conditions of the Creative Commons Attribution (CC BY) license (https://creativecommons.org/licenses/by/4.0/).

\section{Introduction}

Hashimoto's thyroiditis (HT) is an autoimmune condition characterized by lymphocytic infiltration of the thyroid gland and an increase in serum levels of thyroperoxidase antibodies (TPOAb). Patients complain of constant pain and a foreign body sensation on the front of the neck, sore throat, dry cough, hoarseness. The consequence of this pathological process is hypothyroidism, which is manifested by loss of appetite, chilliness, fatigue, drowsiness, dry skin, an increase in BMI, hair loss, constipation, and menstrual cycle disorders. At the same time, some patients may still be concerned about joint pain, muscle pain, dry mouth and sclera, severe general fatigue, loss of interest in life, that is, symptoms of HT extra thyroid effects. 
However, hypothyroidism is not the only consequence of HT. In some patients, against the background of lymphocytic infiltration and hyperplasia of lymphoid tissue, the goiter may develop in the form of diffuse enlargement of the thyroid gland (TG) or the development of false and true nodes. In this case, in addition to a cosmetic disadvantage, there may be a compression syndrome with clinical manifestations of compression of the trachea, esophagus, laryngeal nerves, and neck vessels.

The presence of HT in the absence of specific pathogenetically substantiated treatment in the case of euthyroidism provides for follow-up dynamics: once a year, ultrasound of the thyroid gland and every 6 months' control of the level of thyroid-stimulating hormone (TSH) in the blood serum. With the development of hypothyroidism, TG hormone replacement therapy is carried out mainly with thyroxine preparations or a combination of it and triiodothyronine. Under the influence of such treatment, the symptoms of hypothyroidism and local manifestations of HT usually disappear. However, in some patients, mainly with a high content of TPOAb (more than $1000 \mathrm{IU} / \mathrm{ml}$ ) and a TSH level within the reference values, symptoms of HT, hypothyroidism, and extra-thyroid lesions are observed against the background of adequate replacement therapy with thyroxine preparations. About the lives of such patients, I. Guldvog said that the fatigue caused by Hashimoto's disease is depleting. People cannot work. Their quality of life is very poor. Medical treatment does not make it possible to remove all the symptoms. Hashimoto is not a disease to cause your death, but it can make your existence unbearable. In this context, it is worth mentioning another statement made by I. Guldvog at the International Thyroid Congress (2015), "Thyroxine does NOT normalize the level of symptoms or the level of antithyroid peroxidase" [1].

In the literature, these two theses expressed by I. Guldvog are actively discussed, and based on these discussions, certain conclusions can be drawn about such clinical situations. Firstly, against the background of replacement therapy with thyroxine drugs in laboratory euthyroidism, symptoms of hypothyroidism may not disappear. Secondly, there may be signs of autoimmune damage to other organs and systems, which are called 'extrathyroidal', in HT patients with high TPOAb, in addition to local symptoms and hypothyroidism. In the literature, when discussing this thesis, the authors refer to well-controlled studies that show that thyroxine replacement therapy in the case of laboratory euthyroidism has not proved its effectiveness [24]. At the same time, they draw attention to the established fact that thyroid peroxidase can cross-react with tissues other than thyroid and cause their inflammation, which is manifested by various symptoms, in particular pain in the joints and muscles, dry mouth and sclera, fatigue, and disorders psyche (apathy), decreased memory, etc. (extrathyroidal symptoms of HT).

Given the results of these studies, it can be assumed that Hashimoto's thyroiditis is a specific local manifestation of general autoimmune dysfunction in the body, and against its background, thyroid peroxidase plays the role of a trigger for the development of autoimmune aggression in other organs and systems. Serum TPOAb can cause a cross-reaction with other tissues. This is supported by TPOAb detected in the synovial fluid of HT patients with concomitant polyarthritis. According to the results of many studies, lymphocytes producing activated thyroid antibodies can leave the thyroid gland and penetrate into other distant tissue, causing inflammation and the development of extrathyroid symptoms in it [5-7]. It was also found that the level of TPOAb decreases in parallel with a decrease in the content of other immunological mediators of T-lymphocytes, pro-inflammatory cytokines, interferon-gamma, and tumor necrosis factor-alpha [8-13]. Thus, if HT patients have extrathyroid symptoms on the background of adequate replacement therapy with thyroxin, then to improve their condition 
or reduce the intensity of clinical manifestations, the elimination of the active autoimmune process in TG will be a pathogenetically substantiated action. Since there are no methods of drug exposure to this process, the surgical method remains the method of choice - the complete removal of the autoimmune altered thyroid gland.

In 2019, the use of the surgical method in the treatment of HT patients, and its results were reported by I. Guldvog et al. [14]. This publication caused a lively discussion, mainly with positive reviews, but with caution regarding the possible increase in cases of specific intraoperative complications, in particular injuries of the recurrent nerves and parathyroid glands with corresponding consequences due to the technical difficulties of performing thyroidectomy at HT [15-20].

In discussion papers, in particular, it is written that in the general context HT patients treatment, the issue of indications for surgical treatment, and the choice of the volume of surgery deserves special attention.

As for the indications for the surgical treatment of HT, we have analyzed the works dedicated to this issue over the past 20 years. So, A. F. Carli et al. [21] recommended thyroidectomy as the treatment of choice for surgical treatment of HT, especially when combined with differentiated carcinoma. The presence of compression syndrome and cosmetic deficiency on the front of the neck in HT patients is considered an absolute indication for the surgery [22-25]. Yin C. Kon et al. [26] performed surgical treatment of HT with severe persistent pain in the anterior surface of the neck against the background of treatment with thyroxine. Specialists from the Clayman Thyroid Center believe that surgical treatment of HT is indicated in the presence of compression syndrome, as well as when the drug treatment does not relieve pain on the anterior surface of the neck [27].

Summing the data from these publications, it can be noted that there are no clearly defined, pathogenetically or clinically substantiated indications, and, in general, the issue of surgical treatment of HT is treated with great caution.

Obviously, the choice of a treatment method for HT should be approached based on clearly defined positions, for example, the classification of this autoimmune disease, which would reflect the presence of goiter, its structure, the hormonal function of the gland, the level of antibodies to thyroperoxidase and the features of clinical manifestations of the disease. The literature uses the classification of P. Caturegli et al. (2014) [28] that is based on the clinical forms of TC and structural changes in the gland. There is a classification of I. I. Dedov and G. A. Melnichenko [29], which is not fundamentally different from the previous one. We use wellknown criteria for evaluating the features of the clinical course of this disease, which we have grouped and improved in order to select treatment tactics for patients with HT:

- Hashimoto's thyroiditis without goiter, euthyroidism.

- Hashimoto's thyroiditis without goiter, hypothyroidism.

- Hashimoto's thyroiditis with goiter without compression syndrome, euthyroidism or hypothyroidism.

- Hashimoto's thyroiditis with goiter, compression syndrome, euthyroidism or hypothyroidism.

- Hashimoto's thyroiditis with or without goiter, TPOAb level of more than $1000 \mathrm{IU} / \mathrm{ml}$, hypothyroidism with extrathyroidal clinical symptoms.

In accordance with this distribution of patients, the tactics and methods of treatment were chosen: option 1 - dynamic monitoring, including monitoring of TSH level once every six months and ultrasound of the gland once a year; option 2 - controlled replacement therapy 
with thyroxine and ultrasound of the gland once a year; option 3 - with a nodular transformation of the gland, a fine-needle aspiration puncture biopsy is necessary and only in case of obtaining a cytological conclusion according to the classification of Bethesda IV-VI, surgical treatment is indicated; in the clinical course of HT, which corresponds to option 4 and 5, we consider it appropriate to use surgical treatment.

The feasibility and validity of surgical treatment of HT in the clinical course of goiter with extrathyroidal symptoms today is the subject of active discussion, which was triggered by the aforementioned publication I. Guldvog et al. [14]. The authors presented the results of assessing the quality of life of HT patients according to the SF-36 questionnaire, in particular, patients after thyroidectomy and replacement therapy compared with patients who underwent only standard thyroid hormone therapy. They found that after 18 months, the quality of life indicators of people treated with the same method (thyroidectomy) improved: the average overall health index increased with a difference between groups of 29 points $(p<0.001)$, the fatigue score decreased with a difference between groups of 9.3 points, and the frequency of chronic fatigue between groups decreased from $85 \%$ to $39 \%$. The authors observed infectious complications (in 3 patients), prolonged hypocalcemia (in 3 cases), and unilateral transient laryngeal nerve palsy (in 4 cases) as surgical complications. The level of antibodies to peroxidase decreased on average from $2232 \mathrm{IU} / \mathrm{ml}$ before surgery to $152 \mathrm{IU} / \mathrm{ml}$ after it.

In conclusion, the authors noted that thyroidectomy with thyroxine replacement therapy leads to a significant increase in quality of life indicators compared to patients who were treated with drugs. They believe that the decrease in the severity of clinical signs of hypothyroidism and extrathyroidal manifestations of HT is due to the removal of thyroid antigen as sources of autoimmune aggression, normalization of the level of TPOAb titer and inflammatory mediators in the blood serum.

Long-term observations of HT patients became the motivation for this study. The analysis of the clinical course with local and extrathyroidal symptoms and the ineffectiveness of drug treatment suggested the idea of surgical treatment of this disease in order to eliminate the active autoimmune process in the body - autoimmune thyroiditis. Moreover, we proceeded from the current level of knowledge about autoimmune diseases. The results of the analysis of the clinical course of HT indicate that at the onset of the disease it develops as organ-specific, and subsequently, for some reasons, acquires signs of a systemic one, and, according to the classification of autoimmune diseases, it can be attributed to intermediate ones [30,31]. In this case, the organ-specific autoimmune process provokes the development of systemic disease and supports it. Thus, we can hope that after eliminating the root cause, that is, HT, the clinical manifestations of systemic autoimmune aggression will decrease.

The objective of the work was to study the effect of thyroidectomy on the quality of life of patients with HT with extrathyroidal manifestations.

\section{Materials and Methods}

We examined 37 with HT patients whounderwent surgical treatment in the Ternopil City Emergency Hospital from January 2008 to March 2020. The long-term results of treatment with an assessment of the quality of life were studied in 32 patients 2 years after surgery (the main group). The treatment results were compared with the results of the control group that included 29 HT patients who underwent drug treatment of hypothyroidism with thyroxine preparations with control of the TSH level within euthyroidism. 
A total of 61 women aged 23 to 45 years $(35.20 \pm 0.61)$ were examined in a state of euthyroidism at a dose of thyroxine replacement therapy from 112.5 to $225.0 \mu \mathrm{g} / \mathrm{day}$. The duration of HT disease before inclusion in the study ranged from 7 to 19 years $(11.80 \pm 0.53)$, and treatment with thyroxine - from 2 to 8 years $(4.20 \pm 0.32)$. In terms of average indicators, the age of the patients, the duration of the disease, and the dose of thyroxine replacement therapy received did not differ significantly in both groups.

The criteria for inclusion in the research were:

1. Patient's consent to the study.

2. The presence of HT with hypothyroidism compensated by thyroxine preparations and severe extrathyroidal symptoms.

3. Absence of common somatic and systemic autoimmune diseases.

4. The level of TPOAb in the blood is more than $1000 \mathrm{IU} / \mathrm{ml}$.

Before inclusion in the study, all patients were examined for thyroid function, the level of TPOAb in the blood, the structure of the gland was evaluated using ultrasound, the nodules present in 4 patients were benign according to the results of a fine-needle aspiration biopsy.

Thyroidectomy was performed using an extrafascial technique under intravenous anesthesia with respiratory support. To monitor the larynx nerves, we used the technology we had developed for identifying the larynx nerves among the tissues of the surgical wound [32]. Treatment in the hospital lasted 2-3 days. Complications of surgical treatment were: wound site infiltrate -1 ; hypoparathyroidism -3 (all transient); transient unilateral paresis of the larynx -2 .

The quality of life of patients in both groups was determined upon inclusion in the study and after 2 years of observation. We chose the term arbitrarily after 2 years for re-examination in order to determine the quality of life. Moreover, we believed that the studied parameters for such a period of time could be reliably normalized or stably change in response to the treatment applied. To evaluate it, we used tests of the SF-36 questionnaire, which includes 36 questions combined in 8 scales [33-35]. According to the scales, we studied the indicators of physical functioning $(\mathrm{PF})$ of role functioning due to the physical condition (Role-physical functioning, $\mathrm{RF}$ ) of pain intensity (Bodily pain, BP) of general health (GH) of vital activity (Vitality, VT) social functioning (SF) role-based functioning due to the emotional state (Role emotional, $\mathrm{RE}$ ) mental health $(\mathrm{MH})$. We analyzed the responses of patients that they entered in the SF-36 questionnaire personally during the control examination. The obtained results were compared with indicators before taking for the study.

The study regimen for patients of both groups was as follows: when they were included in the study, patients filled out the SF-36 questionnaire, the levels of TSH and TPOAb were determined in them; every six months, the level of TSH was determined and, if necessary, dose adjustment of replacement therapy and ultrasound were performed; after 2 years they were examined repeatedly - patients filled out a questionnaire, the levels of TSH and TPOAb were determined in them. Patients in the main group were monitored for TSH levels 2 months after surgery and, if appropriate, changed the dose of thyroxine. In both groups, the dose of thyroxine replacement therapy within the limits of euthyroidism was controlled during the observation. In case of a change in dose, its adequacy was checked after 2 months.

\section{Results and Discussion}

Processing of the initial data showed that the average values of the quality of life indicators of patients of both groups were identical and significantly differed from the level of 
ideal health (Tables 1,2). A detailed analysis of the quality of life revealed that the indicators of the psych emotional sphere and physical functioning were most significantly impaired in patients of both groups at the time of inclusion in the study. The results of the study of quality of life indicators are shown in Tables 1 and 2.

Table 1. Quality of life indicators after drug treatment.

\begin{tabular}{l|l|l}
\multirow{2}{*}{ STUDIED INDICATOR } & \multicolumn{2}{|l}{ Drug treatment $(\mathrm{n}=29)$} \\
\cline { 2 - 3 } & before & after 2 years \\
\hline Physical functioning & $49.4 \pm 0.87$ & $49.1 \pm 0.85$ \\
\hline Role limitations due to physical health & $36.7 \pm 2.24$ & $35.9 \pm 2.23$ \\
\hline Pain intensity & $32.1 \pm 1.08$ & $31.2 \pm 1.04$ \\
\hline General health perceptions & $19.8 \pm 0.69$ & $19.1 \pm 0.65$ \\
\hline Vitality & $20.3 \pm 1.18$ & $19.7 \pm 1.18$ \\
\hline Social functioning & $38.4 \pm 1.52$ & $37.2 \pm 1.48$ \\
\hline Role limitations due to emotional problems & $46.4 \pm 2.91$ & $44.4 \pm 2.81$ \\
\hline Mental health & $42.6 \pm 0.55$ & $41.4 \pm 0.49$ \\
\hline TPOAb, IU/ml & $1791.7 \pm 89.17$ & $1584.5 \pm 101.16$ \\
Note. Changes are not reliable $(\mathrm{p}>0.05)$. & &
\end{tabular}

\subsection{Results of drug treatment.}

Drug treatment and this is mainly replacement therapy for hypothyroidism with thyroxine drugs did not affect the overall quality of life of patients with HT during 2 years of use (see Table 1). Six patients with HT duration of 15 years and more indicated a deterioration in the quality of life indicators, such as social functioning and role functioning, due to emotional state. We believe that such an assessment of the quality of life is logical, given that it reflects their psycho-emotional state, due to the progression of the disease and the ineffectiveness of the treatment received. Serum TPOAb values decreased in most patients but remained high. These changes are not statistically reliable.

Table 2. Quality of life indicators after surgical treatment.

\begin{tabular}{l|l|l}
\multirow{2}{*}{ STUDIED INDICATOR } & \multicolumn{2}{|l}{ Surgical treatment $(\mathrm{n}=32)$} \\
\cline { 2 - 3 } & before & after 2 years \\
\hline Physical functioning & $49.8 \pm 0.87$ & $87.7 \pm 0.67$ \\
\hline Role limitations due to physical health & $33.6 \pm 2.24$ & $75.8 \pm 2.91$ \\
\hline Pain intensity & $30.9 \pm 1.10$ & $84.5 \pm 1.42$ \\
\hline General health perceptions & $18.7 \pm 0.59$ & $70.3 \pm 0.45$ \\
\hline Vitality & $19.3 \pm 1.16$ & $76.2 \pm 0.64$ \\
\hline Social functioning & $35.4 \pm 1.47$ & $83.9 \pm 1.84$ \\
\hline Role limitations due to emotional problems & $43.2 \pm 2.88$ & $77.8 \pm 3.85$ \\
\hline Mental health & $41.2 \pm 0.35$ & $84.8 \pm 0.61$ \\
\hline $\begin{array}{l}\text { TPOAb, IU/ml } \\
\text { Note. Changes are reliable }(\mathrm{p}<0.001) .\end{array}$ & $1866.3 \pm 120.24$ & $79.9 \pm 7.59$
\end{tabular}

\subsection{Results of surgical treatment.}

The studied indicators of physical and psych emotional assessment of the quality of life significantly and reliably improved in patients treated with the surgical method after 2 years (see Table 2). The improvement of individual indicators ranged from 34 (role-based functioning due to emotional state) to $57 \%$ (vitality), and the indicator of general health increased by $52 \%$. There were no responses in any questionnaire that indicated an increase in the intensity of individual symptoms. TPOAb titer values decreased almost to the level of physiological values and in the group as a whole were 1.5 times higher.

Why TPOAb is determined in the blood after removal of the thyroid gland is difficult to explain, because their specificity indicates that the body has thyroid tissue. 
Obviously, the way it is. Ideally, it is almost impossible to perform thyroidectomy and, moreover, under conditions of HT and a marked adhesion process against the background of inflammation, without leaving invisible particles of the TG. The scintigraphy of the thyroid gland bed after thyroidectomy for cancer in almost every case, regardless of the stage of the tumor process, shows areas of isotope accumulation. Therefore, these residues of thyroid tissue and are the source of TPOAb formation, and their presence after thyroidectomy for HT is a marker of the thyroidectomy usefulness.

We share the opinion of I. Guldvog et al. [14] on the mechanisms of the effectiveness of HT surgical treatment. The authors substantiate their findings with the results of previous studies regarding the mechanisms of arthritis in HT patients. This concerns the participation of other immunological mediators in TPOAb inflammation, such as T-lymphocytes, interferongamma, tumor necrosis factor-alpha, interleukins 1 and 2, and C-reactive protein [8-13].

\section{Conclusions}

Thyroidectomy in patients with Hashimoto's thyroiditis with local and extrathyroidal symptoms against the background of drug euthyroidism can improve the quality of life in general and for each of the studied parameters in particular. The level of antibodies to thyroperoxidase after surgical treatment is reduced to almost physiologically significant indicators. Finding out the reason for improving the quality of life and the role of reducing serum levels of antibodies to thyroid peroxidase is one of the directions for revealing the pathogenetic mechanisms of extrathyroidal complications of Hashimoto's thyroiditis. The drug treatment of hypothyroidism based on Hashimoto's thyroiditis with local and extrathyroidal symptoms does not improve the quality of life of patients.

\section{Funding}

This research received no external funding.

\section{Acknowledgments}

This research has no acknowledgment.

\section{Conflicts of Interest}

The authors declare that all the procedures and experiments of this study respect the ethical standards in the Helsinki Declaration of 1975, as revised in 2008(5), as well as the national law.

\section{References}

1. Nenkov, R.; Radev, R.; Khristozov, K.; Kuzmanov, I.; Kornovski, S.; Kuzmanov, S.; Krasnaliev, I.; Nanev, B. Hashimoto's thyroiditis: indications for surgical treatment. Khirurgiia (Sofiia) 2005, 28-32.

2. Petnehazy, E.; Buchinger, W. Hashimoto Thyreoiditis, therapeutische Optionen und extrathyreoidale Assoziationen - ein aktueller Überblick. Wiener Medizinische Wochenschrift 2020, 170, 26-34, https://doi.org/10.1007/s10354-019-0691-1.

3. Ott, J.; Promberger, R.; Kober, F.; Neuhold, N.; Tea, M.; Johannes, H.; Hermann, M. Hashimoto's Thyroiditis Affects Symptom Load and Quality of Life Unrelated to Hypothyroidism: A Prospective CaseControl Study in Women Undergoing Thyroidectomy for Benign Goiter. Thyroid : official journal of the American Thyroid Association 2011, 21, 161-167, https://doi.org/10.1089/thy.2010.0191. 
4. Angell, T.E. Thyroidectomy Improves Quality of Life and Fatigue in Patients with Hashimoto's Disease and Persistent Symptoms Compared to Adequate Thyroid Hormone Replacement. Clinical Thyroidology 2019, 31, 178-181, https://doi.org/10.1089/ct.2019;31.178-181.

5. Punzi, L.; Betterle, C. Chronic autoimmune thyroiditis and rheumatic manifestations. Joint Bone Spine 2004, 71, 275-283, https://doi.org/10.1016/j.jbspin.2003.06.005.

6. Punzi, L.; Schiavon, F.; Ramonda, R.; Cavasin, F.; Ruffatti, A.; Todesco, S. Anti-thyroid microsomal antibody in synovial fluid as a revealing feature of seronegative autoimmune thyroiditis. Clin Rheumatol 1991, 10, 181-183, https://doi.org/10.1007/bf02207661.

7. Weetman, A.P.; McGregor, A.M.; Lazarus, J.H.; Hall, R. Thyroid antibodies are produced by thyroidderived lymphocytes. Clin Exp Immunol 1982, 48, 196-200.

8. Karanikas, G.; Schuetz, M.; Wahl, K.; Paul, M.; Kontur, S.; Pietschmann, P.; Kletter, K.; Dudczak, R.; Willheim, M. Relation of anti-TPO autoantibody titre and T-lymphocyte cytokine production patterns in Hashimoto's thyroiditis. Clin Endocrinol (Oxf) 2005, 63, 191-196, https://doi.org/10.1111/j.13652265.2005.02324.x.

9. Norheim, K.B.; Jonsson, G.; Omdal, R. Biological mechanisms of chronic fatigue. Rheumatology (Oxford) 2011, 50, 1009-1018, https://doi.org/10.1093/rheumatology/keq454.

10. Omdal, R.; Brede, C.; Hjelle, A.; Tjensvoll, A.B.; Norheim, K.; Bårdsen, K.; Jonsdottir, K.; Ruoff, P.; Nilsen, M.M. A proteomic signature of fatigue in primary Sjogren's syndrome. Ann. Rheumatic Diseases 2017, 76, 184-185, http://dx.doi.org/10.1136/annrheumdis-2017-eular.3973.

11. Bårdsen, K.; Nilsen, M.M.; Kvaløy, J.T.; Norheim, K.B.; Jonsson, G.; Omdal, R. Heat shock proteins and chronic fatigue in primary Sjögren's syndrome. Innate Immun 2016, 22, 162-167, https://doi.org/10.1177/1753425916633236.

12. Montoya, J.G.; Holmes, T.H.; Anderson, J.N.; Maecker, H.T.; Rosenberg-Hasson, Y.; Valencia, I.J.; Chu, L.; Younger, J.W.; Tato, C.M.; Davis, M.M. Cytokine signature associated with disease severity in chronic fatigue syndrome patients. Proceedings of the National Academy of Sciences 2017, 114, E7150-E7158, https://doi.org/10.1073/pnas.1710519114.

13. Krysiak, R.; Okopien, B. The Effect of Levothyroxine and Selenomethionine on Lymphocyte and Monocyte Cytokine Release in Women with Hashimoto's Thyroiditis. The Journal of Clinical Endocrinology \& Metabolism 2011, 96, 2206-2215, https://doi.org/10.1210/jc.2010-2986.

14. Guldvog, I.; Reitsma, L.C.; Johnsen, L.; Lauzike, A.; Gibbs, C.; Carlsen, E.; Lende, T.H.; Narvestad, J.K.; Omdal, R.; Kvaløy, J.T.; Hoff, G.; Bernklev, T.; Søiland, H. Thyroidectomy Versus Medical Management for Euthyroid Patients With Hashimoto Disease and Persisting Symptoms: A Randomized Trial. Ann Intern Med 2019, 170, 453-464, https://doi.org/10.7326/m18-0284.

15. Schwarz, E.B. Thyroidectomy: An Effective Approach to Hashimoto Disease. NEJM J. Watch 2019.

16. Hui, C.; Karhade, D.S. Thyroidectomy more effective than medication for Hashimoto disease with persisting symptoms. 2 Minute Medicine 2019.

17. Helse Stavanger, H.F. Hashimoto - a Surgical Disease. Absolute Total Thyroidectomy Makes Antibodies Disappear and Ameliorates Symptoms [Electronic resource] URL: https://clinicaltrials.gov/ct2/show/results/NCT02319538.

18. Young, K. Thyroidectomy Seems to Improve Quality of Life in Hashimoto Disease. NEJM J. Watch 2019, 12.

19. Garber, J.R.; Cobin, R.; Gharib, H.; Hennessey, J.; Klein, I.; Mechanick, J.; Pessah-Pollack, R.; Singer, P.; Woeber, K. American Association of Clinical Endocrinologists and American Thyroid Association Taskforce on Hypothyroidism in Adults. Clinical practice guidelines for hypothyroidism in adults: Cosponsored by the American Association of Clinical Endocrinologists and the American Thyroid Association. Endocrine Practice: Official Journal of the American College of Endocrinology and the American Association of Clinical Endocrinologists 2012, 18, 988-1028, https://doi.org/10.4158/EP12280.GL.

20. Sharma, V. Thyroid surgery for patients with Hashimoto's disease. Clin. Thyroidology Public 2019, 12, 8-9.

21. Carli, A.F.; Lottini, M.; Testa, M.; Neri, A. Surgical treatment of Hashimoto's thyroiditis. Personal experience. Minerva Chir 2002, 57, 117-122.

22. Shimizu, K.; Nakajima, Y.; Kitagawa, W.; Akasu, H.; Takatsu, K.; Ishii, R.; Tanaka, S. Surgical therapy in Hashimoto's thyroiditis. J Nippon Med Sch 2003, 70, 34-39, https://doi.org/10.1272/jnms.70.34.

23. McManus, C.; Luo, J.; Sippel, R.; Chen, H. Should patients with symptomatic Hashimoto's thyroiditis pursue surgery? J Surg Res 2011, 170, 52-55, https://doi.org/10.1016/j.jss.2011.01.037.

24. Foppiani L, Secondo V, Arlandini A, Quilici P, Cabria M, Del Monte P. Thyroid lymphoma: a rare tumor requiring combined management. Hormones (Athens) 2009;8:214-8.Pradeep, P.V.; Ragavan, M.; Ramakrishna, B.A.; Jayasree, B.; Skandha, S.H. Surgery in Hashimoto's thyroiditis: indications, complications, and associated cancers. J Postgrad Med 2011, 57, 120-122, https://doi.org/10.4103/00223859.81867 . 
25. Kon, Y.C.; DeGroot, L.J. Painful Hashimoto's thyroiditis as an indication for thyroidectomy: clinical characteristics and outcome in seven patients. J Clin Endocrinol Metab 2003, 88, 2667-2672, https://doi.org/10.1210/jc.2002-021498.

26. Rashmi Roy. Hashimoto's Thyroiditis [Electronic resource]. 2020-URL: https://www.thyroidcancer.com/hashimotos-thyroiditis.

27. Caturegli, P.; De Remigis, A.; Rose, N.R. Hashimoto thyroiditis: clinical and diagnostic criteria. Autoimmun Rev 2014, 13, 391-397, https://doi.org/10.1016/j.autrev.2014.01.007.

28. Dedov II, Melnichenko GA, Popov AS. Endocrinology in art. Thyroid disease: A history with geography in pictures and charts. Volume 1. Moscow: ROOI People's Health, 2015; pp. 64.

29. Fridkis-Hareli, M. Immunogenetic mechanisms for the coexistence of organ-specific and systemic autoimmune diseases. J Autoimmune Dis 2008, 5, 1-1, https://doi.org/10.1186/1740-2557-5-1.

30. Eaton, W.W.; Rose, N.R.; Kalaydjian, A.; Pedersen, M.G.; Mortensen, P.B. Epidemiology of autoimmune diseases in Denmark. J Autoimmun 2007, 29, 1-9, https://doi.org/10.1016/j.jaut.2007.05.002.

31. Shidlovskyi, O.; Sheremet, M.; Shidlovskyi, V.; Bedenyuk, A.; Maksymyuk, V.; Tkachuk, N.; Bezruk, V.; Bezruk, T.; Batig, V.M.; Chympoi, K.; Gresko, M.D. Electrophysiological identification of nerves of the larynx among the tissues of operative wound in goiter surgeries. Archives of the Balkan Medical Union 2017, $52,408-413$.

32. Sheremet, M.I.; Sydorchuk, L.P.; Shidlovskyi, V.O.; Bedenyuk, A.D. Research of prognostic markers of proliferation and apoptosis in patients with nodular goiters combined with autoimmune thyroiditis. Archives of the Balkan Medical Union 2016, 51, 488-491.

33. Loge, J.H.; Ekeberg, O.; Kaasa, S. Fatigue in the general Norwegian population: normative data and associations. J Psychosom Res 1998, 45, 53-65, https://doi.org/10.1016/s0022-3999(97)00291-2.

34. HÅvard Loge, J.; Kaasa, S. Short Form 36 (SF-36) health survey: normative data from the general Norwegian population. Scandinavian Journal of Social Medicine 1998, 26, 250-258, https://doi.org/10.1177/14034948980260040401. 